http://jmscr.igmpublication.org/home/ ISSN (e)-2347-176x ISSN (p) 2455-0450

crossref DOI: https://dx.doi.org/10.18535/jmscr/v10i2.17

Journal Of Medical Science And Clinical Research

\title{
The role of Antibiogram in the evaluation of Anti-Microbial Stewardship Program in present South India
}

\begin{abstract}
Authors
Sheeba K Thomas ${ }^{1}$, Shoba Kurian Pulikottil ${ }^{2}$

${ }^{1}$ Assistant Professor, Department of Microbiology, Government Medical College, Kottayam, Kerala, India

${ }^{2}$ Professor and Head, Department of Microbiology, Government Medical College, Kottayam, Kerala, India

Abstract

Even though there are opinions that the antibiogram is intrinsically flawed and it needs to address multiple confounders, nevertheless the general consensus in that the antibiogram continues to remain as a consistent measurable unit of the effectiveness of Anti-Microbial Stewardship Programs in contributing to patient care. Antimicrobial resistance was predicted even by Alexander Flemming himself to Penicillin. Therefore, almost in every centre, the measures and strategies in antimicrobial stewardship program address primarily how to decrease the problems created by antibiotic resistance. The experience with trying to decrease the use of fluoroquinolones following rampant resistance only resulted in increased use of and subsequent resistance to Carbapenems. This phenomenon of squeezing the balloon' ought not to be repeated. Although all agree that appropriate antibiotic use definitely brings down financial burden on the patient, how far it helps in actually bringing down the length of hospital is still not clear. Measuring the conservable days of therapy is supposed to give a clearer picture. It is also agreed that the rate of hospital acquired infection by multiple drug resistant organisms is more useful than calculating the proportion of resistant isolates. Finally, the important recommendation for India is to implement a uniform hospital antibiotic policy and ensure faithful adherence. There is a need to agree on a uniform method of antibiotic susceptibility testing in all hospital microbiology laboratories that also includes testing of resistance of higher drugs. Rapid PCR testing of MRSA versus MSSA (methicillin resistant versus sensitive Staphylococcus aureus) would be useful in blood culture. Quality control and assurance programs need to be set up.

Keywords: AMSP Antimicrobial Stewardship Program, AMR Antimicrobial resistance, Antibiogram.
\end{abstract}

\section{Background}

A review of literature in India and abroad reveal that though all aspects of the role of Antibiogram in AMSP have been discussed for and against, nevertheless, the Antibiogram alone continues to exist as a consistent measurable unit of the effectiveness of AMSP in contributing to patient care.
Alexander Flemming, who discovered the first antibiotic Penicillin, predicted emergence of resistance to the same in the coming years. Selective pressure of antibiotics makes bacteria evolve and acquire resistance eventually.

Therefore, isn't AMR a natural phenomenon? or is AMR seriously due to irrational use of antibiotics? Recent history of microbiology and therapeutics in dealing with HIV-AIDS, Hepatitis B, Hepatitis C, 
Viral influenza and the ongoing Covid crisis indicates that generally all hospital personnel including doctors are willing to adhere to evidence based protocol and policies. This can be extrapolated to AMSP as well.

\section{Squeezing the Balloon}

Following widespread propaganda against the use of Ciprofloxacin (Fluoroquinolones) and increasing resistance to it, its use has remarkably decreased and consequently so has resistance against it. However, the actual impact was that it resulted in increased use of Meropenem and Imipenem and consequent resistance against it. This phenomenon is called 'squeezing the balloon' and needless to say, this cannot be viewed as an ideal AMSP intervention.

\section{Current Scenario}

There is an ocean of data on AMSP both in India and abroad but not as many studies commensurate with this volume of data. There are useful contributions from Centre for Disease Control CDC, Clinical and Laboratory Standards Institute CLSI, Infectious Diseases Society of America IDSA, Society for Healthcare Epidemiology of America SHEA, Indian Council of Medical Research ICMR and Indian Academy of Pediatrics. Often, more than analysis, critical appraisal is what really helps in reaching a conclusion.

The National Nososcomial Infections Surveillance report in 2004 draws a direct association between increasing rates of hospital-acquired infections versus increased costs, adverse events, antimicrobial resistance and super-infections. Similar such studies and subsequent reports prompted the AMSPs. This has led to standard guidelines on particularly the prevention of HAI by Clostridium difficile. In order to effectively decrease the incidence of Clostridium difficile infections, the AMSP intervention is to decrease the unnecessary and inappropriate use of antibiotics rather than the total use.
Tina MK et al in her article has elaborately described various studies and how in few cases, it did help in bringing down resistance to certain higher antibiotics. However, she emphatically argues that for AMSP pertaining to Community acquired pneumonia, skin and soft tissue infections, sepsis and bloodstream infections, there are five quality metrics which include:

1) Days of drug therapy

2) Number of patients with specific MDR organisms

3) Mortality caused by MDRO

4) Conservable days of therapy and not length of stay

5) Unplanned hospital readmission within thirty days of discharge

As AMR increased, the AMSP intervention isn't actually limiting the quantity of antibiotics but the target is the quality and appropriateness of antibiotic use. Antibiotic use can be used to evaluate the quality of patient care, similar to other AMSP interventions such as Surgical Prophylaxis. As part of Infection Prevention and Control, there are seven measures and three include the selection, timing and discontinuation of perioperative antibiotic prophylaxis.

Lucas TS et al in his article on the actual role of Antibiogram in AMSP states that the Antibiogram is intrinsically flawed and that there are many confounders. The confounders include the presence of comorbidities, secular trends, resistance outbreaks, concomitant infection prevention strategies and in India, alternatives in treatment options, particularly Ayurvedic or Homeo treatment prior to hospitalization. There is also a strong opinion among certain authors that when relating antibiotic use to the emerging resistance, the rate calculated is a better indicator that proportion.

The ICMR recommendation on AMSP included participation by departments of Pharmacology and presently, they are already actively involved in efforts that include documentation of adverse events and toxicity and on how to minimize these. As a result, certain centres attempted antibiotic 
cycling protocol with fluoroquinolones and betalactams as empiric therapy every 3 months for all infections except meningitis, endocarditis and sexually transmitted infections. In certain places, it helped in bringing down aminoglycoside related nephrotoxicity.

Regarding collateral damage of antibiotic use in the assessment of patient care, there is a general consensus that AMSP should address other outcomes such as clinical success or failure of the particular antibiotic, conservable days of therapy as opposed to length of hospital stay and infection-related morbidity and mortality.

\section{Recommendations}

At least in India, the most urgent need of the hour is to formulate a uniform hospital antibiotic policy and ensure that it is adhered to faithfully.

Second is to ensure that all microbiology laboratories follow a uniform pattern of antibiotic susceptibility methods that address drugs that cover Gram positives, Gram negatives and broad spectrum. Even though, certain centres perform additional tests to trace ESBLs, AmpC and Carbapenemases, this also needs a uniform procedure. As far as Gram positives are concerned, it's prudent to use a rapid PCR test to differentiate between Meticillin resistant Staphylococcus aureus-MRSA and Methicillin sensitive Staphylococcus aureus-MSSA, whenever isolated from blood culture.

It would be prudent to have programs for assuring quality control and quality assurance for all microbiology laboratories on a state level and national level.

\section{Conflict of Interest: Nil}

Acknowledgement: This study was done as part of State Board of Medical Research Project funded by Government of Kerala.

\section{References}

1. Sujith J Chandy et al. ICMR programme on Antibiotic Stewardship, Prevention of
Infection \& Control (ASPIC).Indian J Med Res. 2014 Feb; 139(2): 226-230.

2. KaminiWalia et al. Policy document on antimicrobial stewardship practices in India. Indian J Med Res. 2019 Feb; 149(2): 180-184.

3. World Health Organization. The World Health Report 2008 - Primary Health Care (Now More Than Ever), The challenges of a changing world. Figure 8 . The shift towards non-communicable diseases and accidents as causes of death. [accessed on January 3, 2012]. http://www.who.int/whr/2008/08_chap1_fi g08_en.pdf.

4. Sheeba K Thomas et al. Phenotype Prevalence of ESBLs and Genotype Prevalence of CTX-M from respiratory infections in tertiary care centre in central Kerala. JMSCR Vol 6 Issue 9 Sept 2018. 06:368-373.

5. Sheeba K Thoams et al. Phenotype and Genotype Prevalence of Carbapenemase genes in bacterial isolates from lower respiratory tract infections in a tertiary care centre in central Kerala. JMSCR Vol 7 Issue 9 Sept 2019. 07:549-553.

6. Sheeba K Thomas et al. Detection of Carbapenem resistance by Carbapenem Inactivation Method in tertiary care centre in central Kerala. JMSCR Vol 9 Issue 10: 111-115. Oct 2021

7. Tina MK et al. Antimicrobial Stewardship: A Matter of Process or Outcome? Pharmacotherapy 2012; 32(8): 688-706.

8. Lucas TS et al. Can the Antibiogram be used to Assess Microbiologic Outcomes after Antimicrobial Stewardship Interventions? A Critical Review of the Literature. Pharmacotherapy 2012; 32(8): 668-676.

9. Gales $\mathrm{AC}$ et al. Characterization of Pseudomonas aeruginosa isolates: occurrence rates, antimicrobial susceptibility patterns, and molecular 
typing in the global SENTRY

Antimicrobial Surveillance Program, 1997-1999. Clin Infect Dis. 2001;32(Suppl 2):S146-5.

10. Hoa NQet al. Decreased Streptococcus pneumoniae susceptibility to oral antibiotics among children in rural Vietnam: a community study. BMC Infect Dis. 2010;10:85.

11. Mathai E, Chandy SJ et al. Antimicrobial resistance surveillance among commensal Escherichia coli in rural and urban areas in Southern India. Trop Med Int Health. 2008; 13:41-5.

12. NidhiBedi, Piyush Gupta. Antimicrobial Stewardship in Pediatrics: An Indian Perspective. Indian Pediatr 2016;53: 293298. 